PROCEEDINGS OF THE

AMERICAN MATHEMATICAL SOCIETY

Volume 136, Number 10, October 2008, Pages 3611-3620

S 0002-9939(08)09368-4

Article electronically published on June 2, 2008

\title{
BANACH SPACES WITH A UNIQUE NONTRIVIAL DECOMPOSITION
}

\author{
SPIROS A. ARGYROS AND THEOCHARIS RAIKOFTSALIS
}

(Communicated by N. Tomczak-Jaegermann)

\begin{abstract}
Motivated by a problem of P. Koszmider we introduce the class of quasi-prime Banach spaces. This class lies between the classes of prime and primary Banach spaces. It is shown that for every $1<p<\infty$ there exists a strictly quasi-prime separable reflexive Banach space $\mathfrak{X}_{p}$ such that $\ell_{p}$ is a complemented subspace of $\mathfrak{X}_{p}$. A similar result also holds for the case of $\ell_{1}$ and $c_{0}$. More generally, for every separable decomposable prime Banach space $Y$ not containing $\ell_{1}$ there exists a strictly quasi-prime $\mathfrak{X}_{Y}$ containing $Y$ as a complemented subspace. We also investigate the operators acting on these spaces as well as the complemented subspaces of their finite powers.
\end{abstract}

\section{INTRODUCTION}

A Banach space $X$ is said to be prime if it is isomorphic to each one of its infinite dimensional complemented subspaces. A classical result of A. Pelczynski 12 yields that $c_{0}$ and $\ell_{p}$ for $1 \leq p<\infty$ are all prime spaces, and J. Lindenstrauss in 9] has also shown that $\ell_{\infty}$ shares the same property. More recently, W.T. Gowers and B. Maurey [7] have shown that there exists an indecomposable reflexive prime Banach space not containing any $\ell_{p}$ for $1 \leq p<\infty$. It remains open whether the only decomposable (i.e. spaces which are the direct sum of two infinite dimensional subspaces) separable prime Banach spaces are the known ones. In particular, it is not known if mixed Tsirelson spaces with a subsymmetric basis, like Schlumprecht's space [13, could be prime.

A wider class is that of primary Banach spaces, namely spaces $X$ with the property that whenever $X \cong Y \oplus Z$ then either $Z \cong X$ or $Y \cong X$. As is well known, classical results yield that this class includes $L^{p}(0,1), C[0,1]$, James space $J$, James Tree space $J T$ and others.

In a recent paper P. Koszmider 8, under $\mathrm{CH}$, has constructed a nonseparable $C(K)$ space satisfying the property that whenever $C(K) \cong Y \oplus Z$ then either $Y \cong c_{0}$ and $Z \cong C(K)$ or vice versa. In the same paper he also asked whether a separable Banach space could occur sharing similar properties. This problem was also mentioned by Y. Benyamini in his review for P. Koszmider's paper.

Received by the editors July 12, 2007, and, in revised form, September 11, 2007.

2000 Mathematics Subject Classification. Primary 46B20, 46B26.

Key words and phrases. Prime Banach space, primary Banach space, Schauder decomposition, hereditarily indecomposable Banach space, strictly singular operators, complemented subspaces.

This work was partially supported by the Leukippos NTUA Research programme. 
In the present note we answer affirmatively P. Koszmider's question. In particular, we introduce a new class of primary Banach spaces called quasi-prime. Their definition is motivated by the properties of Koszmider's space and can be stated as follows: A Banach space $\mathfrak{X}$ is said to be quasi-prime if there exists an infinite dimensional subspace $Y$ of $\mathfrak{X}$ such that $\mathfrak{X}$ admits a unique nontrivial decomposition as $\mathrm{Y} \oplus \mathfrak{X}$. If $\mathrm{Y}$ is isomorphic to $\mathfrak{X}$, then $\mathfrak{X}$ is prime; otherwise $\mathfrak{X}$ will be called strictly quasi-prime. As is shown every strictly quasi-prime space has exactly two, up to isomorphism, complemented subspaces, and more importantly the space admits a unique nontrivial decomposition. Our main results concerning strictly quasi-prime spaces are included in the next theorem.

Theorem I. The following hold:

(1) For every $p \geq 1$ there exists a separable Banach space $\mathfrak{X}_{p}$ which is strictly quasi-prime containing $\ell_{p}$ as a complemented subspace. Moreover, for every $p>1$ the corresponding space is reflexive.

(2) There exists a strictly quasi-prime separable Banach space $\mathfrak{X}_{0}$ containing $c_{0}$ (as a complemented subspace).

(3) Let $Y$ be a separable decomposable prime Banach space not containing $\ell_{1}$. Then there exists a separable strictly quasi-prime Banach space $\mathfrak{X}_{Y}$ which contains $Y$ as a complemented subspace.

Each $\mathfrak{X}_{p}$ is the HI Schauder sum of the corresponding $\ell_{p}$ and similarly for $\mathfrak{X}_{0}$. The external HI norm is based on the Gowers-Maurey norm as in [2. The space $\mathfrak{X}_{Y}$, in the third part of the theorem above, is also an HI Schauder sum of the space $Y$ where the external norm uses higher complexity saturation methods as in 5. The proof of the theorem above is presented in sections 2 and 3 . In section 4 for $\mathfrak{X}$ a Schauder sum of a sequence of spaces $\left(X_{n},\|\cdot\|\right)_{n}$ we define the class of horizontally strictly singular operators $T: \mathfrak{X} \rightarrow \mathfrak{X}$, and we prove the following:

Theorem II. Let $\mathfrak{X}=\left(\sum_{n=1}^{\infty} \oplus X_{n}\right)_{\text {gm }}$ be an HI Schauder sum of a sequence $\left(X_{n}\right.$, $\left.\|\cdot\|_{n}\right)_{n}$ of separable Banach spaces. Assume that for every $\left(w_{n}\right)_{n}$ (horizontal) block sequence in $\mathfrak{X}$ the space $W=\overline{\left\langle w_{n}: n \in \mathbb{N}\right\rangle}$ is totally incomparable to each $X_{n}$. Then for every $T: \mathfrak{X} \mapsto \mathfrak{X}$ bounded linear operator there exists $\lambda \in \mathbb{R}$ such that $T-\lambda I$ is horizontally strictly singular.

We also prove that for $n \neq m$ the spaces $\mathfrak{X}_{p}^{n}, \mathfrak{X}_{p}^{m}$ are not isomorphic and similarly for $\mathfrak{X}_{0}$. This yields that for every $n \in \mathbb{N}$ the space $\mathfrak{X}_{p}^{n}$ has at least $n+1$ pairwise nonisomorphic complemented subspaces. We extend our thanks to P. Wojtaszczyk and the referee for their valuable comments and remarks.

\section{The SPACES $\mathfrak{X}_{p}$ FOR $1 \leq p<\infty$ AND $\mathfrak{X}_{0}$}

Notation. Let $\left(X_{n},\|\cdot\|_{n}\right)_{n}$ be a sequence of separable Banach spaces. Then

i. By $\Omega$ we denote the cartesian product $\Omega=\prod_{n=1}^{\infty} X_{n}$.

ii. We denote the elements of $\Omega$ by $\tilde{x}, \tilde{y}$, etc. For a given vector $\tilde{x} \in \Omega$ we denote by $\operatorname{supp} \tilde{x}$ the set $\operatorname{supp} \tilde{x}=\{n \in \mathbb{N}: \tilde{x}(n) \neq 0\}$. We set $\Omega_{00}=\{\tilde{x} \in \Omega:|\operatorname{supp} \tilde{x}|<\infty\}$ and $\Omega_{00}^{*}=\left\{\tilde{x}^{*} \in \prod_{n=1}^{\infty} X_{n}^{*}:\left|\operatorname{supp} \tilde{x}^{*}\right|<\infty\right\}$.

iii. For $\tilde{x} \in \Omega_{00}$, by $\operatorname{ran} \tilde{x}$ we denote the minimal interval of the natural numbers $[\min \operatorname{supp} \tilde{x}, \max \operatorname{supp} \tilde{x}]$ that contains $\operatorname{supp} \tilde{x}\left(\operatorname{ran} \tilde{x}^{*}\right.$ has an analogous meaning).

iv. For two subsets $E_{1}, E_{2}$ of $\mathbb{N}$ we write $E_{1}<E_{2}$ if $\max E_{1}<\min E_{2}$. For $\tilde{x}, \tilde{y} \in \Omega_{00}$ we write $\tilde{x}<\tilde{y}$ whenever $\operatorname{supp} \tilde{x}<\operatorname{supp} \tilde{y}$. A sequence $\left(\tilde{x}_{n}\right)_{n}$ in $\Omega_{00}$ is said to be block if $\tilde{x}_{n}<\tilde{x}_{n+1}$ for all $n \in \mathbb{N}$. 
v. For every $A \subset \mathbb{N}$ we denote by $P_{A}$ the linear map $P_{A}: \Omega \mapsto \Omega$ with

$$
P_{A}(\tilde{x})(k)=\tilde{x}(k) \text { if } k \in A \text { and } 0 \text { otherwise. }
$$

Additionally, for $A=\{1, \ldots, n\}$ we denote $P_{A}$ by $P_{[1, n]}$ and $I-P_{A}$ by $P_{(n, \infty)}$. Also for simplicity we set $P_{\{n\}}=P_{n}$, for $n \in \mathbb{N}$.

Finally, for $k \in \mathbb{N}$ we denote by $i_{k}: X_{k} \mapsto \Omega_{00}$ the natural embedding.

Definition 1. A Banach space $(\tilde{X},\|\cdot\|)$ is called a Schauder sum of the sequence $\left(X_{n},\|\cdot\|_{n}\right)_{n}$ if the following are satisfied:

i. $\Omega_{00} \subseteq \tilde{X}$ and $\Omega_{00}$ is dense in $\tilde{X}$.

ii. $\|x\|_{n}=\left\|i_{n}(x)\right\|$, for every $n \in \mathbb{N}$ and $x \in X_{n}$.

iii. For every $n \in \mathbb{N}$ and $\tilde{x} \in \tilde{X}, P_{[1, n]}: \tilde{X} \mapsto \tilde{X}$ is bounded and $\tilde{x}=$ $\lim _{n \in \mathbb{N}} P_{[1, n]}(\tilde{x})$.

iv. A Schauder sum $\tilde{X}$ of the sequence $\left(X_{n},\|\cdot\|_{n}\right)_{n}$ will be called bimonotone if for every interval $I$ of $\mathbb{N}$ we have that $\left\|P_{I}(\tilde{x})\right\| \leq\|\tilde{x}\|$ for every $\tilde{x} \in \tilde{X}$.

v. A Schauder sum $\tilde{X}$ will be called boundedly complete if for any $\tilde{x} \in \Omega$ the relation $\sup _{n \in \mathbb{N}}\left\|P_{[1, n]}(\tilde{x})\right\|<\infty$ implies $\tilde{x} \in \tilde{X}$.

vi. A Schauder sum $\tilde{X}$ will be called shrinking if for any $\tilde{x}^{*} \in \tilde{X}^{*}$ we have $\tilde{x}^{*}=\lim _{n} P_{[1, n]}^{*}\left(\tilde{x}^{*}\right)$, where $P_{[1, n]}^{*} \tilde{x}^{*}(\tilde{x})=\tilde{x}^{*}\left(P_{[1, n]}(\tilde{x})\right)$ for $\tilde{x} \in \tilde{X}$. This yields that the space $\tilde{X}^{*}$ is a Schauder sum of the sequence $\left(X_{n}^{*}\right)_{n}$.

We note that if a Schauder sum $\tilde{X}$ of a sequence $\left(X_{n},\|\cdot\|_{n}\right)_{n}$ is boundedly complete and shrinking, then $\tilde{X}$ is reflexive if and only if each $X_{n}$ is reflexive. In 2] it was shown that given a sequence of separable Banach spaces $\left(X_{n},\|\cdot\|_{n}\right)_{n}$ there exists a Gowers-Maurey type HI Schauder sum of the sequence $\left(X_{n},\|\cdot\|_{n}\right)_{n}$. Namely, we have the following

Theorem 1. Let $\left(X_{n},\|\cdot\|_{n}\right)_{n}$ be a sequence of separable Banach spaces. Then there exists a Banach space $\mathfrak{X}=\left(\sum_{n=1}^{\infty} \oplus X_{n}\right)_{\text {gm }}$ satisfying the following properties:

1. This space $\mathfrak{X}$ is a Schauder sum of the sequence $\left(X_{n},\|\cdot\|_{n}\right)_{n}$. In addition, $\mathfrak{X}$ is boundedly complete and shrinking. Thus, if every $X_{n}$ is reflexive, then $\mathfrak{X}$ is reflexive, too.

2. By the shrinking property of $\mathfrak{X}$ we have that each block sequence in $\mathfrak{X}$ is weakly null.

3. Every block sequence $\left(\tilde{x}_{n}\right)_{n}$ generates a HI subspace; i.e. the subspace $\overline{\left\langle\tilde{x}_{n}: n \in \mathbb{N}\right\rangle}$ of $\mathfrak{X}$ is $H I$.

4. For a finite block sequence $\left(f_{i}\right)_{i=1}^{n}$ in $B_{\mathfrak{X}^{*}}$ we have that $\frac{1}{\log _{2}(n+1)} \sum_{i=1}^{n} f_{i} \in B_{\mathfrak{X}^{*}}$

We give the definition of a quasi-prime Banach space.

Definition 2. A Banach space $X$ is said to be quasi-prime if there exists a subspace $\mathrm{Y}$ of $X$ such that $X$ admits a unique nontrivial decomposition as $\mathrm{Y} \oplus X$. If $\mathrm{Y}$ is not isomorphic to $X$, then $X$ will be called strictly quasi-prime.

Remark 1. It is worth indicating the connection of a decomposable prime or quasiprime Banach space to its finite codimensional subspaces. Namely we have

1. If $X$ is a prime Banach space such that $X$ admits a unique nontrivial decomposition as $X \cong X \oplus X$, then $X$ is isomorphic to all its finite codimensional subspaces. We present a short argument showing this. Let $Z$ be a closed hyperplane of $X$; then

$$
X \cong X \oplus X \cong X \oplus(\mathbb{R} \oplus Z) \cong(X \oplus \mathbb{R}) \oplus Z
$$


This yields that $X \cong Z$. Using induction it is easy to check that $X$ is isomorphic to all its finite codimensional subspaces. The above also yields that if $X$ is quasi-prime space (see Definition 2) and $Y$ is isomorphic to $X$, then $X$ is prime.

2. Let $X$ be a quasi-prime Banach space admitting a unique nontrivial decomposition as $X \cong X \oplus Y$ where $Y$ is not isomorphic to $X$. One of the following alternatives holds: Either $Y \cong Z$ whenever $\operatorname{codim}(Z)=2 n-1, n \in \mathbb{N}$, and $X \cong Z$ whenever $\operatorname{codim}(Z)=2 n, n \in \mathbb{N}$ or $X$ is isomorphic to all its finite codimensional subspaces. To see this first note that $X \cong X \oplus Y \cong X \oplus(Y \oplus Y)$ which yields that $Y \oplus Y \cong Y$. Now let $Z$ be a closed hyperplane of $X$; then $X \oplus Y \cong Z \oplus(Y \oplus \mathbb{R})$. Hence, we have two cases.

Either $Z \cong X$ and $Y \oplus \mathbb{R} \cong Y$ or $Z \cong Y$ and $Y \oplus \mathbb{R} \cong X$. The first one shows that $X$ is isomorphic to all of its finite codimensional subspaces. As for the second we have the following:

$$
X \oplus X \cong X \oplus Y \oplus X \oplus Y \cong Y \oplus(Y \oplus \mathbb{R}) \oplus Y \oplus(Y \oplus \mathbb{R}) \cong Y \oplus \mathbb{R}^{2} .
$$

However $X \oplus X$ is not isomorphic to $X$, and thus if $W$ is a subspace of $X$ with codimension 2, we have $X \cong X \oplus Y \cong\left(Y \oplus \mathbb{R}^{2}\right) \oplus W$. Thus $X$ must be isomorphic to $W$. This analysis gives us that in the second case we have that $X$ must be isomorphic to its subspaces of even codimension and $Y$ isomorphic to its subspaces of odd codimension.

Let us point out that all the examples of decomposable quasi-prime spaces presented in this paper fall into the first alternative stated in the the above remark; namely they are all isomorphic to their finite codimensional subspaces. We do not know if there exists a quasi-prime not isomorphic to its hyperplanes.

We now pass to prove the existence of separable strictly quasi-prime Banach spaces. In the sequel we shall denote by $\mathfrak{X}_{0}$ the Gowers-Maurey HI Schauder sum of the sequence $X_{n}=c_{0}$ for all $n \in \mathbb{N}$, and for $p \geq 1$ we set $\mathfrak{X}_{p}$ the corresponding one of the sequence $X_{n}=\ell_{p}$ for all $n \in \mathbb{N}$; our intention is to show that these spaces are strictly quasi-prime. Let's observe that the space $\mathfrak{X}_{p}$ has as a complemented subspace the space $\ell_{p}$, and it is not an isomorphic $\ell_{p}$ since $\mathfrak{X}_{p}$ contains HI subspaces. Similarly for $\mathfrak{X}_{0}$. Therefore, neither $\mathfrak{X}_{p}$ nor $\mathfrak{X}_{0}$ is a prime space. Our intention is to prove that the above spaces are indeed strictly quasi-prime. We start with some preliminary lemmas.

Lemma 1. Let $\mathfrak{X}=\mathfrak{X}_{p}$ or $\mathfrak{X}=\mathfrak{X}_{0}$ and suppose that $\mathfrak{X}=Y \oplus Z$. Then neither $Y$ nor $Z$ contain an HI subspace.

Proof. Assume the contrary and let $Y^{\prime}, Z^{\prime}$ be HI subspaces of $Y$ and $Z$, respectively. Since $Y$ and $Z$ are complements of $\mathfrak{X}$ there exists $\epsilon>0$ such that $d\left(S_{Y}, S_{Z}\right)>\epsilon$. As $Y^{\prime}, Z^{\prime}$ are HI we have that for every $k \in \mathbb{N}$ the maps $P_{[1, k]} \mid Y^{\prime}: Y^{\prime} \mapsto \sum_{n=1}^{k} X_{n}$ and $P_{[1, k]} \mid Z^{\prime}: Z^{\prime} \mapsto \sum_{n=1}^{k} X_{n}$ are both strictly singular. Thus, using a sliding hump argument we may construct a block sequence $\left(w_{n}\right)_{n}$ in $\mathfrak{X}$ and normalized sequences $\left(y_{n}\right)_{n},\left(z_{n}\right)_{n}$ in $Y^{\prime}$ and $Z^{\prime}$, respectively, such that $\left\|y_{n}-w_{2 n}\right\|<\delta_{2 n}$ and $\left\|z_{n}-w_{2 n+1}\right\|<\delta_{2 n+1}$ for a sequence $\left(\delta_{n}\right)_{n}$ of positive numbers such that $\sum_{n=1}^{\infty} \delta_{n}<\frac{\epsilon}{4}$. By Theorem 11 we have that if we set $W_{1}=\overline{\left\langle w_{2 n}: n \in \mathbb{N}\right\rangle}$ and $W_{2}=\overline{\left\langle w_{2 n+1}: n \in \mathbb{N}\right\rangle}$, we have $d\left(S_{W_{1}}, S_{W_{2}}\right)=0$, which implies that

$$
d\left(S_{Y}, S_{Z}\right) \leq d\left(S_{Y^{\prime}}, S_{Z^{\prime}}\right)<\frac{\epsilon}{2},
$$

and this is a contradiction. 
Lemma 2. Let $Y$ be a subspace of $\mathfrak{X}$, where $\mathfrak{X}=\mathfrak{X}_{p}$ or $\mathfrak{X}=\mathfrak{X}_{0}$, not containing an $H I$ subspace. Then there exists a $k_{0} \in \mathbb{N}$ such that the map $P_{\left[1, k_{0}\right]}$ restricted on $Y$ is an isomorphic embedding.

Proof. Assume the contrary. Then for every $k \in \mathbb{N}$ the map $P_{[1, k]} \mid Y$ is not an isomorphism. Thus for every $k \in \mathbb{N}$ and $\epsilon>0$ there exists a $y \in Y$ with $\|y\|=1$ such that $\left\|P_{[1, k]}(y)\right\|<\epsilon$. Thus using a sliding hump argument we construct a normalized block sequence $\left(z_{n}\right)_{n}$ and a sequence $y_{n} \in Y$ such that $\sum_{n}\left\|y_{n}-z_{n}\right\|<\frac{1}{4}$. Hence by Theorem 1 the space $\overline{\left\langle z_{n}: n \in \mathbb{N}\right\rangle}$ is $\mathrm{HI}$ and isomorphic to the subspace of $Y$ generated by $\left(y_{n}\right)_{n}$, and this leads to a contradiction.

Lemma 3. Let $Y$ be a subspace of $\mathfrak{X}$, where $\mathfrak{X}=\mathfrak{X}_{p}$ or $\mathfrak{X}=\mathfrak{X}_{0}$, not containing an HI subspace. We distinguish the following cases:

1. If $p=1$ and $Y$ is complemented in $\mathfrak{X}$ with projection $P: \mathfrak{X} \mapsto Y$, then for every $\epsilon>0$ there exists $k_{\epsilon} \in \mathbb{N}$ such that for every $x \in \sum_{i=k_{\epsilon}+1}^{\infty} X_{i}$ we have $\|P(x)\|<\epsilon\|x\|$.

2. If $p>1$ or $\mathfrak{X}=\mathfrak{X}_{0}$, then for every $\epsilon>0$ there exists a $k_{\epsilon} \in \mathbb{N}$ such that $\left\|P_{\left(k_{\epsilon}, \infty\right)}(y)\right\|<\epsilon\|y\|$ for all $y \in Y$.

Proof. The case of $\ell_{1}$.

Assume not. Then there exists an $\epsilon>0$ such that for every $k \in \mathbb{N}$ there exists $x_{k} \in \mathfrak{X}_{(k, \infty)}$ such that $\left\|P\left(x_{k}\right)\right\|>\epsilon$. Thus, using a sliding hump argument we may assume that that $\left(x_{k}\right)_{k}$ is a block. As $\left(x_{k}\right)_{k}$ is block we have that $\left(x_{k}\right)_{k}$ is weakly null and thus $P\left(x_{k}\right)_{k}$ is weakly null. On the other hand, Lemma 2 yields that $Y$ is isomorphic to a subspace of $\ell_{1}$, and by the Schur property of $\ell_{1}$ we have that $\left\|P\left(x_{k}\right)\right\| \stackrel{k}{\rightarrow} 0$. Contradiction.

The case of $\ell_{p}$ for $p>1$ and $c_{0}$.

We present a proof only in the case of $\ell_{p}$, as the arguments in the case of $c_{0}$ are almost identical. Consider $p>1$ and assume that the conclusion fails. Then there exists a normalized sequence $\left(y_{k}\right)_{k}$ in $Y$ and a sequence $\left(I_{k}\right)_{k}$ of successive intervals of $\mathbb{N}$ such that for the maps $P_{I_{k}}: \mathfrak{X}_{p} \mapsto \sum_{i \in I_{k}} X_{i}$ we have $\left\|P_{I_{k}}\left(y_{k}\right)\right\| \geq \epsilon$. For every $k \in \mathbb{N}$ choose a functional $f_{k}$ in the norming set $\mathcal{G}$ such that $\operatorname{ran} f_{k} \subset I_{k}$ and $f_{k}\left(P_{I_{k}}\left(y_{k}\right)\right)>\epsilon$. Since by Lemma 2 we know that $Y$ is isomorphic to a subspace of $\ell_{p}$ we may assume, by passing to a subsequence if necessary, that the sequence $z_{k}=y_{2 k}-y_{2 k-1}$ is weakly null and by passing to a further subsequence we have that $\left(z_{k}\right)_{k}$ is equivalent to the standard basis of $\ell_{p}$. Hence, there exists a $C>0$ such that for all $n \in \mathbb{N}$ we have $\left\|\frac{1}{n} \sum_{i=1}^{n_{l}} z_{i}\right\| \leq C \frac{n^{1 / p}}{n}$. We can choose $n \in \mathbb{N}$ such that $C \frac{n^{1 / p}}{n}<\frac{\epsilon}{\log _{2}(n+1)}$ as $\frac{\log _{2}(n+1)}{n^{(p-1) / p}} \rightarrow 0$. At the same time we have that the functional $f=\frac{1}{\log _{2}(n+1)} \sum_{i=1}^{n} f_{i}$ belongs to the norming set $\mathcal{G}$, and this gives us $f\left(\frac{1}{n} \sum_{i=1}^{n} z_{i}\right) \geq \frac{\epsilon}{\log _{2}(n+1)}$, which is a contradiction.

Proposition 1. Let $\mathfrak{X}$ denote either $\mathfrak{X}_{p}, 1 \leq p<\infty$ or $\mathfrak{X}_{0}$. Also let $Y$ be a complemented subspace of $\mathfrak{X}, Z$ be its complement, and assume that both $Y$ and $Z$ are infinite dimensional. Then either $Y \cong \ell_{p}$ (respectively $c_{0}$ ) and $Z \cong \mathfrak{X}_{p}$ (respectively $\mathfrak{X}_{0}$ ) or vice versa.

Proof. By Lemma 1 we can assume that $Y$ does not contain an HI subspace. Let $P: \mathfrak{X} \rightarrow Y$ be a projection of $\mathfrak{X}$ onto $Y$. First we shall show that there exists a $k \in \mathbb{N}$ such that $Y$ is isomorphic to a complemented subspace of $\sum_{i=1}^{k} X_{i}$. As 
$\sum_{i=1}^{k} X_{i}$ is isomorphic to $\ell_{p}$ or $c_{0}$ for any $k \in \mathbb{N}$ by a classical result of Pelczynski, this would imply that $Y \cong \ell_{p}$ or $Y \cong c_{0}$. We will only treat the case of $\ell_{p}$, as the proof for $c_{0}$ goes through identical arguments.

By Lemma 3 we can choose $k \in \mathbb{N}$ such that

$$
\left\|\left.P P_{(k, \infty)}\right|_{Y}\right\|<1 \text { and }\left\|\left.P_{(k, \infty)} P\right|_{\mathfrak{x}_{(k, \infty)}}\right\|<1 .
$$

Taking into account the identities

(2) $I_{Y}-P P_{[1, k]}=\left.P P_{(k, \infty)}\right|_{Y}$ and $I_{\mathfrak{X}_{(k, \infty)}}-\left.P_{(k, \infty)}(I-P)\right|_{\mathfrak{X}_{(k, \infty)}}=\left.P_{(k, \infty)} P\right|_{\mathfrak{X}_{(k, \infty)}}$

we deduce that the operators

$$
\left.P P_{[1, k]}\right|_{Y}: Y \rightarrow Y,\left.P_{(k, \infty)}(I-P)\right|_{\mathfrak{X}_{(k, \infty)}}: \mathfrak{X}_{(k, \infty)} \rightarrow \mathfrak{X}_{(k, \infty)}
$$

are both invertible. Therefore, the operators $P_{[1, k]}: Y \rightarrow P_{[1, k]}(Y)$ and $\left.P\right|_{P_{[1, k]}(Y)}$ : $P_{[1, k]}(Y) \rightarrow Y$ are invertible as well. This yields $Y \cong P_{[1, k]}(Y)$. In addition, denoting by $T$ the operator $\left.P_{[1, k]} P\right|_{P_{[1, k]}(Y)}: P_{[1, k]}(Y) \rightarrow P_{[1, k]}(Y)$, we can see that $T$ is also invertible. Let $\iota$ be the natural inclusion of $P_{[1, k]}(Y)$ into $\sum_{i=1}^{k} X_{i}$. Then we have the following factorization:

$$
P_{[1, k]}(Y) \stackrel{\iota}{\longrightarrow} \sum_{i=1}^{k} X_{i} \stackrel{\left.P_{[1, k]} P\right|_{\sum_{i=1}^{k} X_{i}}}{\longrightarrow} P_{[1, k]}(Y)
$$

which yields that the operator

$$
\left.T^{-1} P_{[1, k]} P\right|_{\sum_{i=1}^{k} X_{i}}: \sum_{i=1}^{k} X_{i} \rightarrow P_{[1, k]}(Y)
$$

is a projection of $\sum_{i=1}^{k} X_{i}$ onto $P_{[1, k]}(Y)$. Hence, $Y$ is isomorphic to a complemented subspace of $\sum_{i=1}^{k} X_{i}$ and therefore $Y \cong \ell_{p}$.

We will now show that $Z \cong \mathfrak{X}_{p}$. Using similar arguments as in the case of $Y$, we see that the factorization of the invertible operator $\left.P_{(k, \infty)}(I-P)\right|_{\mathfrak{X}_{(k, \infty)}}$ : $\mathfrak{X}_{(k, \infty)} \rightarrow \mathfrak{X}_{(k, \infty)}$ given by $\mathfrak{X}_{(k, \infty)} \stackrel{\left.(I-P)\right|_{\mathfrak{X}_{(k, \infty)}} ^{\rightarrow}}{\rightarrow} \stackrel{P_{(k, \infty)}}{\rightarrow} \mathfrak{X}_{(k, \infty)}$ yields that $\mathfrak{X}_{(k, \infty)}$ is isomorphic to a complemented subspace of $Z$. In particular, $\ell_{p}$ is isomorphic to a complemented subspace of $Z$. In order to see that $Z \cong \mathfrak{X}_{p}$ we write

$$
Z \cong \ell_{p} \oplus W \cong \ell_{p} \oplus \ell_{p} \oplus W \cong \ell_{p} \oplus Z \cong \mathfrak{X}_{p},
$$

and this completes the proof.

All the above yields the next theorem.

Theorem 2. The following hold:

1. For every $p \geq 1$ the space $\mathfrak{X}_{p}$ is not isomorphic to $\ell_{p}$, and it has exactly two complemented infinite dimensional subspaces, namely $\ell_{p}$ and $\mathfrak{X}_{p}$ itself, and the only decomposition of $\mathfrak{X}_{p}$ into two infinite dimensional subspaces $i s \ell_{p} \oplus \mathfrak{X}_{p}$. In addition, if $p>1$, then $\mathfrak{X}_{p}$ is reflexive.

2. The space $\mathfrak{X}_{0}$ is not isomorphic to $c_{o}$, and it has exactly two complemented infinite dimensional subspaces, namely $c_{0}$ and $\mathfrak{X}_{0}$ itself, and the only decomposition of $\mathfrak{X}_{0}$ into two infinite dimensional subspaces is $c_{0} \oplus \mathfrak{X}_{0}$.

In particular the space $\mathfrak{X}_{0}$ provides an affirmative answer to P. Koszmider's question [8]. 


\section{Quasi-PRIMe HI Schauder sums of A PRIME SPACE $Y$}

In this section we start with an arbitrary separable prime and decomposable space $Y$, which does not contain an isomorphic copy of $\ell_{1}$, and we provide an example of a quasi-prime $\mathfrak{X}_{Y}$ not isomorphic to $Y$ such that $\mathfrak{X}_{Y}=\mathfrak{X}_{Y} \oplus Y$. The construction of $\mathfrak{X}_{Y}$ uses higher complexity HI Schauder sums and relies heavily on earlier results concerning the Schreier hierarchy $\left(\mathcal{S}_{\xi}\right)_{\xi<\omega_{1}}$ introduced in [1] and the repeated averages hierarchy $R A$ developed in $[3$. For the results used we refer the reader to [5] for a detailed exposition. We start with the following result form [3]

Lemma 4. Let $Y$ be a separable Banach space that does not contain $\ell_{1}$. Then there exists a countable ordinal $\xi<\omega_{1}$ such that for every weakly null sequence $\left(y_{n}\right)_{n}$ and every $\epsilon>0$ there exists $L \in[\mathbb{N}]$ such that for every $x^{*} \in B_{Y^{*}}$ we have

$$
\left\{n \in L:\left|x^{*}\left(y_{n}\right)\right|>\epsilon\right\} \in \mathcal{S}_{\xi} .
$$

For a proof we refer to Lemmas 13.32, 13.34 and Proposition 13.35 in [5].

Now let $Y$ be a separable prime space that does not contain $\ell_{1}$. We fix $\xi<\omega_{1}$ such that the conclusion of the previous lemma holds for $Y$. Using an increasing sequence of countable ordinals $\left(\xi_{j}\right)_{j}$ such that $\xi<\xi_{1}$, we construct $\mathfrak{X}_{Y}=$ $\left(\sum_{n=1}^{\infty} Y\right)_{\xi-h i}$, a $\xi$-HI Schauder sum of $Y$ satisfying the additional property:

For every block sequence $\left(f_{i}\right)_{i=1}^{d}$ of functionals in the norming set $\mathcal{G}_{\xi}$ such that $\left\{\min \operatorname{supp} f_{i}: i \leq d\right\} \in \mathcal{S}_{\xi_{2 j}}$ we have that $\frac{1}{m_{2 j}} \sum_{i=1}^{d} f_{i} \in \mathcal{G}_{\xi}$. In order to prove that $\mathfrak{X}_{Y}$ admits a unique nontrivial decomposition $\mathfrak{X}_{Y}=\mathfrak{X}_{Y} \oplus Y$ we follow the same steps as in the cases of $\mathfrak{X}_{p}$ for $p>1$. The only difference is in Lemma 3 , which can be restated in the following manner:

Lemma 5. Let $Z$ be a subspace of $\mathfrak{X}_{Y}$ with the property that there exists $n \in \mathbb{N}$ such that the projection $P_{[1, n]}$ restricted on $Z$ is an isomorphic embedding. Then for every $\epsilon>0$ there exists a $k_{\epsilon} \in \mathbb{N}$ such that $\left\|P_{\left(k_{\epsilon}, \infty\right)}(z)\right\|<\epsilon\|z\|$ for all $z \in Z$.

The proof follows the same steps as in the proof of Lemma 3 . In this case, however, instead of using averages in $Y$ one uses special convex combinations (see [5]) in order to arrive to the desired contradiction.

Theorem 3. The space $\mathfrak{X}_{Y}$ admits a unique nontrivial decomposition as $\mathfrak{X}_{Y} \cong$ $\mathfrak{X}_{Y} \oplus Y$.

Proof. Let $W, Z$ be two complemented subspaces of $\mathfrak{X}_{Y}$ such that $\mathfrak{X}_{Y} \cong W \oplus Z$ and denote by $P: \mathfrak{X}_{Y} \rightarrow Z$ and $I-P: \mathfrak{X}_{Y} \rightarrow W$ the associated projections. As in Lemma 1 one can show that either $P$ or $I-P$ is not an isomorphism restricted on any block subspace of $\mathfrak{X}_{Y}$. We assume that it is $P$ that satisfies this property. Arguing as in Lemma2 2 it is proved that there exists $k \in \mathbb{N}$ such that $P_{[1, k]}$ restricted on $Z$ is an isomorphic embedding. Invoking Lemma 5 for $\epsilon<\min \left\{\frac{1}{2\|P\|}, \frac{1}{2}\right\}$ we find a $k^{\prime} \in \mathbb{N}$ such that

$$
\left\|P_{\left(k^{\prime}, \infty\right)}(z)\right\|<\epsilon\|z\| \text { for all } z \in Z
$$

and thus proceeding as in Proposition 1 we can show that $Z \cong Y$ and $W \cong \mathfrak{X}$.

\section{The SPACE OF Operators ON AN HI Schauder SUM}

In this section we study the structure of bounded linear operators acting on an HI Schauder sum $\mathfrak{X}$. It is shown, under an additional assumption concerning the block subspaces of $\mathfrak{X}$, that for every $T \in \mathcal{L}(\mathfrak{X})$ there exists $\lambda \in \mathbb{R}$ such that $T-\lambda I$ is horizontally strictly singular. The corresponding definition is given below. 
Definition 3. Let $\mathfrak{X}=\left(\sum_{n=1}^{\infty} \oplus X_{n}\right)_{g m}$ be an HI Schauder sum of the spaces $\left(X_{n}\right.$, $\left.\|\cdot\|_{n}\right)_{n}$. A bounded linear operator $S: \mathfrak{X} \mapsto \mathfrak{X}$ will be called horizontally strictly singular if $S$ restricted on an arbitrary block subspace of $\mathfrak{X}$ is not an isomorphism.

We recall the definition of $\ell_{1}$ averages.

Definition 4. Let $k \in \mathbb{N}, M>0$. We call a vector $x \in \mathfrak{X}$ an $M-\ell_{1}^{k}$ average if $\|x\| \geq 1$ and there exists a block sequence $\left(x_{i}\right)_{i=1}^{k}$ with $\left\|x_{i}\right\| \leq M$, for all $i=1, \ldots, k$ such that $x=\frac{1}{k} \sum_{i=1}^{k} x_{i}$.

The following lemma establishes the presence of $2-\ell_{1}^{k}$ averages for every $k \in \mathbb{N}$ in every block subspace of $\mathfrak{X}$. Namely, we have the following:

Lemma 6. Let $Z$ be a block subspace of $\mathfrak{X}$ and $k \in \mathbb{N}$. There there exists a $x \in Z$ which is a $2-\ell_{1}^{k}$ average.

For a proof we refer the interested reader to [4], Lemma II.22.

The next lemma indicates the behavior of a bounded linear operator on a sequence $\left(y_{n}\right)_{n}$ where each $y_{n}$ is a $2-\ell_{1}^{k}$ average with $k \rightarrow \infty$. Its proof is essentially an adaptation of Lemma IV.11 in 4, so we omit it.

Lemma 7. Let $Y$ be block subspace of $\mathfrak{X}$ and $T: Y \rightarrow \mathfrak{X}$ a bounded linear operator. Suppose that $\left(y_{n}\right)_{n}$ is a block sequence of $2-\ell_{1}$ averages with increasing lengths in $Y$ such that the sequence $\left(T\left(y_{n}\right)\right)_{n}$ is also block. Then $\lim _{n} d\left(T y_{n}, \mathbb{R} y_{n}\right)=0$.

The above implies the following.

Lemma 8. Let $\mathfrak{X}=\left(\sum_{n=1}^{\infty} \oplus X_{n}\right)_{\text {gm }}$ be an HI Schauder sum of the spaces $\left(X_{n}\right.$, $\left.\|\cdot\|_{n}\right)_{n}$ and $Y$ a block subspace of $\mathfrak{X}$ such that $Y$ is totally incomparable to each $X_{n}$. Then for every bounded linear operator $T: Y \mapsto \mathfrak{X}$ there exists $\lambda \in \mathbb{R}$ such that $T-\lambda I_{Y}$ is strictly singular, where $I_{Y}$ denotes the natural inclusion of $Y$ into $\mathfrak{X}$.

Proof. We assume that $T$ is not strictly singular. Then there exists a block subspace $Z$ generated by a normalized block sequence $\left(z_{n}\right)_{n}$ such that $T$ restricted on $Z$ is an isomorphism. Since $Z$ is totally incomparable to each $X_{n}$, we have that for each $k \in \mathbb{N}$ the operator $P_{[1, k]} T: Z \rightarrow \sum_{i=1}^{k} X_{i}$ is strictly singular. This yields that for every $\epsilon>0$ there exist block sequences $\left(w_{n}\right)_{n},\left(v_{n}\right)_{n}$ such that the following hold:

i. The sequence $\left(w_{n}\right)_{n}$ is normalized, and $\left(w_{n}\right)_{n} \subset\left\langle z_{n}: n \in \mathbb{N}\right\rangle$.

ii. $\sum_{n=1}^{\infty}\left\|T w_{n}-v_{n}\right\|<\epsilon$.

Therefore, for sufficiently small $\epsilon>0$ we may assume that the sequence $\left(T w_{n}\right)_{n}$ is also a block sequence in $\mathfrak{X}$.

By Lemma 6 we may additionally assume that $\left(w_{n}\right)_{n}$ consists of $2-\ell_{1}$ averages with increasing lengths. Applying Lemma 7 we have that there exists an $L \in[\mathbb{N}]$ and a $\lambda \in \mathbb{R}$ such that $\lim _{n \in L}\left\|T\left(w_{n}\right)-\lambda w_{n}\right\|=0$. Moreover, $L$ can be chosen such that $T-\lambda I_{Y} \mid \overline{\left\langle w_{n}: n \in L\right\rangle}$ is a compact operator. The HI property of $Y$ and Proposition 1.2 in [5] yield that $T-\lambda I_{Y}$ is strictly singular.

An immediate consequence of the preceding arguments is the following.

Theorem 4. Let $\mathfrak{X}=\left(\sum_{n=1}^{\infty} \oplus X_{n}\right)_{\text {gm }}$ be an HI Schauder sum of a sequence $\left(X_{n}\right.$, $\left.\|\cdot\|_{n}\right)_{n}$ of separable Banach spaces. Assume that for every $\left(w_{n}\right)_{n}$ (horizontal) block sequence in $\mathfrak{X}$ the space $W=\overline{\left\langle w_{n}: n \in \mathbb{N}\right\rangle}$ is totally incomparable to each $X_{n}$. Then for every $T: \mathfrak{X} \mapsto \mathfrak{X}$ bounded linear operator there exists $\lambda \in \mathbb{R}$ such that $T-\lambda I$ is horizontally strictly singular. 
Proof. Let $Y$ be a block subspace of $\mathfrak{X}$. Then by the previous lemma there exists $\lambda \in \mathbb{R}$ such that the operator $T-\lambda I$ restricted on $Y$ is strictly singular. Let $Z$ be another block subspace; then it is easy to see that $W=\overline{\langle Y \cup Z\rangle}$ is also an HI space. Since $T-\lambda I_{W}$ restricted on $Y$ is strictly singular, Proposition 1.2 in [5] yields that $T-\lambda I$ restricted on $W$ is also strictly singular. Hence, $T-\lambda I$ is a horizontally strictly singular operator.

The above also yields the following:

Proposition 2. Let $\mathfrak{X}$ be the HI Schauder sum of a sequence $\left(X_{n},\|\cdot\|_{n}\right)_{n}$ of separable Banach spaces and $T: \mathfrak{X} \rightarrow Z$ a bounded linear operator. Assume that there exists a subspace $Y$ of $\mathfrak{X}$ such that $T$ restricted on $Y$ is an isomorphism. If for every $n \in \mathbb{N}$ the projection $P_{[1, n]}$ restricted on $Y$ is strictly singular, then there exists $n_{0} \in \mathbb{N}$ such that $T$ restricted on $P_{\left(n_{0}, \infty\right)}(\mathfrak{X})$ is an isomorphism.

Proof. Suppose not. Then for every $n \in \mathbb{N}$ there exists a $w_{n} \in P_{(n, \infty)}(\mathfrak{X})$ such that $\left\|w_{n}\right\|=1$ and $\left\|T w_{n}\right\|<\frac{1}{2^{n}}$. Clearly, we may assume that the sequence $\left(w_{n}\right)_{n}$ is block. Moreover, using a sliding hump argument we can pick a normalized block sequence $\left(v_{n}\right)_{n}$ almost contained in $Y$ such that if we set $V=\overline{\left\langle v_{n}: n \in \mathbb{N}\right\rangle}$ and $W=$ $\overline{\left\langle w_{n}: n \in \mathbb{N}\right\rangle}$ we have that $T$ restricted on $V$ is an isomorphism and $T$ restricted on $W$ is strictly singular. Since the space $U=\overline{\langle V \cup W\rangle}$ is HI, Proposition 1.2 in [5] yields a contradiction, and this completes the proof.

We now will show that for any $1 \leq p<\infty$ and $n \in \mathbb{N}$ the space $\mathfrak{X}_{p}^{n+1}=$ $\sum_{i=1}^{n+1} \oplus \mathfrak{X}_{p}(i)$ is not isomorphic to $\mathfrak{X}^{n}=\sum_{i=1}^{n} \oplus \mathfrak{X}_{p}(i)$. The same holds for $\mathfrak{X}_{0}$. We present a proof only in the case of $\mathfrak{X}_{p}$ as the arguments in the case of $\mathfrak{X}_{0}$ are identical. In the next proposition all the sums $\sum_{i=1}^{k} \oplus \mathfrak{X}_{p}(i), k \in \mathbb{N}$, are endowed with the supremum norm as the external one.

Proposition 3. Let $n \in \mathbb{N}$ and $T: \sum_{i=1}^{n+1} \oplus \mathfrak{X}_{p}(i) \rightarrow \sum_{i=1}^{n} \oplus \mathfrak{X}_{p}(i)$ be a bounded linear operator. Then $T$ cannot be an isomorphism.

Proof. Assume that $T$ is indeed an isomorphic embedding and choose $\theta>0$ such that $\|T x\| \geq \theta$ for all $x \in \mathfrak{X}_{p}^{n+1}$ with $\|x\|=1$. By Theorem 4 , $T$ can be represented as $T=\left(\lambda_{i j} I_{i j}\right)_{i, j}+\left(S_{i j}\right)_{i, j}$ with $1 \leq i \leq n$ and $1 \leq j \leq n+1$, where $I_{i j}$ is the identity $I_{i j}: \mathfrak{X}_{p}(j) \rightarrow \mathfrak{X}_{p}(i)$ and $S_{i j}: \mathfrak{X}_{p}(j) \rightarrow \mathfrak{X}_{p}(i)$ is a horizontally strictly singular operator.

Consider the matrix $\Lambda=\left(\lambda_{i j}\right)_{i j}$ defining a linear operator $\Lambda: \mathbb{R}^{n+1} \rightarrow \mathbb{R}^{n}$. As $\Lambda$ cannot be an isomorphic embedding there exists a vector $\left(a_{i}\right)_{i=1}^{n+1} \in \mathbb{R}^{n+1}$ such that

i. $\Lambda \cdot\left(a_{i}\right)_{i=1}^{n+1}=\mathbf{0} \in \mathbb{R}^{n}$.

ii. $\left|a_{i}\right| \leq 1$ for all $i \leq 1$.

iii. There exists $k \leq n+1$ such that $\left|a_{k}\right|=1$.

Since each $S_{i j}$ is horizontally strictly singular starting with a block subspace $Y$ of $\mathfrak{X}_{p}$ and repeatedly applying Proposition 2.c.4 in [10] for the operators $S_{i j} \mid Y$, we show that for every $\epsilon>0$ there exists $x \in \mathfrak{X}_{p}$ with $\|x\|=1$ such that

$$
\left\|S_{i j}(x)\right\|<\epsilon \text {, for all } 1 \leq i \leq n, 1 \leq j \leq n+1 .
$$


For $\epsilon$ sufficiently small we choose $x \in \mathfrak{X}_{p}$ as above such that setting $\tilde{S}=\left(S_{i j}\right)_{i j}$ and for $1 \leq m \leq n+1, \tilde{x}_{m}=\left(y_{i}^{m}\right)_{i=1}^{n+1} \in \sum_{i=1}^{n+1} \oplus \mathfrak{X}_{p}(i)$ with $y_{m}^{m}=x$ and $y_{i}^{m}=0, i \neq$ $m$, the following holds: $\left\|\tilde{S}\left(\sum_{i=1}^{n+1} a_{i} \tilde{x}_{i}\right)\right\|<\frac{\theta}{2}$. Observe now that $\left\|\sum_{i=1}^{n+1} a_{i} \tilde{x}_{i}\right\|=$ $\max \left|a_{i}\right|=1$ and $\left\|T\left(\sum_{i=1}^{n+1} a_{i} \tilde{x}_{i}\right)\right\|=\left\|\tilde{S}\left(\sum_{i=1}^{n+1} a_{i} \tilde{x}_{i}\right)\right\|<\frac{\theta}{2}$. This contradiction yields the proof.

Corollary 1. Let $n, m \in \mathbb{N}$ with $n \neq m$. Then the space $\mathfrak{X}^{n}$ is not isomorphic to $\mathfrak{X}^{m}$. Moreover, the space $\mathfrak{X}^{n}$ has at least $n+1$, up to isomorphism, complemented subspaces.

The problem whether the space $\mathfrak{X}^{m}$ has exactly $m+1$, up to isomorphism, complemented subspaces remains open.

\section{REFERENCES}

[1] D. Alspach and S.A. Argyros, Complexity of weakly null sequences, Dissertationes Mathematicae, 321(1992), 1-44. MR.1191024 (93j:46014)

[2] S.A. Argyros and V. Felouzis, Interpolating hereditarily indecomposable Banach spaces, Journal AMS, 13(2001), 243-294. MR1750954 (2002b:46021)

[3] S.A. Argyros, S. Merkourakis and A. Tsarpalias, Convex unconditionality and summability of weakly null sequences, Isr. J. Math., 107(1998), 157-193. MR.1658551 (99m:46021)

[4] S.A. Argyros and S. Todorcevic, Ramsey methods in analysis, Birkhäuser, CRM, Barcelona, 2004. MR2145246 (2006e:46015)

[5] S.A. Argyros and A. Tolias, Methods in the theory of hereditarily indecomposable Banach spaces, Memoirs of the AMS, 170(2004), no. 806. MR2053392 (2005f:46022)

[6] W.T. Gowers and B. Maurey, The unconditional basic sequence problem, J. Amer. Math. Soc., 6(1993), no. 4, 851-874. MR1201238 (94k:46021)

[7] W.T. Gowers and B. Maurey, Banach spaces with small spaces of operators, Math. Ann., 307(1997), no. 4, 543-568. MR 1464131 (98g:46018)

[8] P. Koszmider, On decompositions of Banach spaces of continuous functions on Mrowka's spaces, Proc. AMS, 133(2005), 2137-2146. MR2137881(2006a:46030)

[9] J. Lindenstrauss, On complemented subspaces of $m$, Israel J. Math., 5(1967), 153-156. MR0222616 (36:5666)

[10] J. Lindenstrauss and L. Tzafriri, Classical Banach spaces. I and II, Springer, 1996. MR0500056 (58:17766) MR0540367 (81c:46001)

[11] B. Maurey, Banach spaces with few operators, Handbook of the geometry of Banach spaces, Vol. 2, 1247-1297, North-Holland, Amsterdam, 2003. MR.1999196 (2004m:46014)

[12] A. Pelczynski, Projections in certain Banach spaces, Studia Math., 9(1960), 209-228. MR0126145 (23:A3441)

[13] Th. Schlumprecht, An arbitrarily distortable space, Israel J. Math., 76(1991), 81-95. MR:1177333 (93h:46023)

Faculty of Applied Sciences, Department of Mathematics, Zografou Campus, National Technical University of Athens, 157 80, Athens, Greece

E-mail address: sargyros@math.ntua.gr

Faculty of Applied Sciences, Department of Mathematics, Zografou Campus, National Technical University of Athens, 157 80, Athens, Greece

E-mail address: th-raik@hotmail.com 